\title{
Warm gas in protostellar outflows
}

\section{Extremely high-velocity emission jet and outflows from OMC-2/3}

\author{
A. I. Gómez-Ruiz ${ }^{1}$, A. Gusdorf ${ }^{2}$, S. Leurini ${ }^{3}$, K. M. Menten ${ }^{4}$, S. Takahashi ${ }^{5,6,7}$, F. Wyrowski ${ }^{4}$, and R. Güsten ${ }^{4}$ \\ ${ }^{1}$ CONACYT-Instituto Nacional de Astrofísica, Óptica y Electrónica, Luis E. Erro 1, C.P. 72840, Tonantzintla, Puebla, México \\ e-mail: aigomez@inaoep.mx \\ ${ }^{2}$ LERMA, UMR 8112 du CNRS, Observatoire de Paris, École Normale Supérieure, 61 Av. de l'Observatoire, 75014 Paris, France \\ 3 INAF - Osservatorio Astronomico di Cagliari, Via della Scienza 5, 09047 Selargius (CA), Italy \\ ${ }^{4}$ Max-Planck-Institut für Radioastronomie (MPIfR), Auf dem Hügel 69, 53121 Bonn, Germany \\ 5 Joint ALMA Observatory, Alonso de Córdova 3107, Vitacura, Santiago, Chile \\ ${ }^{6}$ NAOJ Chile Observatory, Alonso de Córdova 3788, Oficina 61B, Vitacura, Santiago, Chile \\ 7 Department of Astronomical Science, School of Physical Sciences, SOKENDAI (The Graduate University for Advanced Studies), \\ Mitaka, Tokyo 181-8588, Japan
}

Received 8 May 2014 / Accepted 7 June 2019

\begin{abstract}
Context. OMC-2/3 is one of the nearest embedded cluster-forming regions that includes intermediate-mass protostars at early stages of evolution. A previous $\mathrm{CO}(3-2)$ mapping survey towards this region revealed outflow activity related to sources at different evolutionary phases.

Aims. The present work presents a study of the warm gas in the high-velocity emission from several outflows found in CO (3-2) emission by previous observations, determines their physical conditions, and makes a comparison with previous results in low-mass star-forming regions.

Methods. We used the CHAMP+ heterodyne array on the APEX telescope to map the CO (6-5) and CO (7-6) emission in the OMC-2 FIR 6 and OMC-3 MMS 1-6 regions, and to observe ${ }^{13} \mathrm{CO}(6-5)$ at selected positions. We analyzed these data together with previous $\mathrm{CO}$ (3-2) observations. In addition, we mapped the SiO (5-4) emission in OMC-2 FIR 6.

Results. The CO (6-5) emission was detected in most of the outflow lobes in the mapped regions, while the CO (7-6) was found mostly in the OMC-3 outflows. In the OMC-3 MMS 5 outflow, a previously undetected extremely high-velocity gas was found in CO (6-5). This extremely high-velocity emission arises from the regions close to the central object MMS 5. Radiative transfer models revealed that the high-velocity gas from MMS 5 outflow consists of gas with $n_{\mathrm{H}_{2}}=10^{4}-10^{5} \mathrm{~cm}^{-3}$ and $T>200 \mathrm{~K}$, similar to what is observed in young Class 0 low-mass protostars. For the other outflows, values of $n_{\mathrm{H}_{2}}>10^{4} \mathrm{~cm}^{-3}$ were found.

Conclusions. The physical conditions and kinematic properties of the young intermediate-mass outflows presented here are similar to those found in outflows from Class 0 low-mass objects. Due to their excitation requirements, mid- $J$ CO lines are good tracers of extremely high-velocity gas in young outflows likely related to jets.
\end{abstract}

Key words. stars: formation - ISM: clouds - ISM: jets and outflows - shock waves - ISM: individual objects: OMC-2/3

\section{Introduction}

Intermediate-mass (IM) protostars, i.e. protostars whose mass is in the range from 2 to $8 M_{\odot}$, provide crucial information on the star formation process because they link the low- and highmass star formation (e.g. Crimier et al. 2010). IM protostars share some properties of massive protostars, such as their ability to ionize the surrounding gas, and that they exist in clusters. On the other hand, they present the advantage that they are more numerous and therefore are located at closer distances $(\lesssim 1 \mathrm{kpc})$ than most of the massive star-forming regions. Therefore, they could fill the gap between low- and high-mass star formation studies. Despite their relevance, very little is known about the formation and early evolutionary stages of IM protostars (e.g. class 0-type objects). Although some detailed studies have been

\footnotetext{
^ Based on observations acquired with the Atacama Pathfinder Experiment (APEX). APEX is a collaboration between the Max-PlanckInstitut für Radioastronomie, the European Southern Observatory, and the Onsala Space Observatory.
}

conducted in recent years (e.g., Beltrán 2015; Takahashi \& Ho 2012; Takahashi et al. 2009, 2006; van Kempen et al. 2012; Crimier et al. 2010; Beltrán et al. 2008; Fuente et al. 2007, 2001; Zapata et al. 2004, 2005), little information is available, in particular about outflows from class 0-type sources.

Observations have shown that outflows from IM protostars (hereafter IM outflows) share some characteristics of outflows from low-mass protostars, such as the high collimation factor in early evolutionary phases (Beltrán et al. 2008). On the other hand, IM outflows are, in general, intrinsically more energetic than those driven by low-mass sources (Beltrán et al. 2008; Takahashi et al. 2008). The values of the outflow momentum rate, $F_{\text {out }}$, are higher in IM protostars than in low-mass objects, which may be an indication that IM young stellar objects (YSOs) accrete material faster (at a higher accretion rate) than low-mass ones (Beltrán et al. 2008).

Regarding their physical parameters, such as kinetic temperature $\left(T_{\text {kin }}\right)$ and $\mathrm{H}_{2}$ density $(n)$, only for a few cases have they been determined via observations of more than one $\mathrm{CO}$ 
transition (e.g., Cep-E and NGC 2071: Gómez-Ruiz et al. 2012; Chernin \& Masson 1992; Hatchell et al. 1999). The best studied case is Cep-E, in which a multi-line CO study at high spectral resolution uncovered different excitation components related to the different kinematic structures (Lefloch et al. 2015). In the jet component, the emission from the low $-J$ CO lines (from $J=1-0$ to $J=5-4)$ is dominated by a gas layer with $T_{\text {kin }}=80$ $100 \mathrm{~K}$, and $n=(0.5-1) \times 10^{5} \mathrm{~cm}^{-3}$; while the high $-J$ CO lines (from $J=12-11$ to $J=16-15$ ) trace warmer and denser gas, with $T_{\text {kin }}=400-750 \mathrm{~K}$, and $n=(0.5-1) \times 10^{6} \mathrm{~cm}^{-3}$. In the outflow cavity, the low $-J \mathrm{CO}$ lines are dominated by a gas layer with $T_{\text {kin }}=55-85 \mathrm{~K}$, and density in the range $(1-8) \times 10^{5} \mathrm{~cm}^{-3}$; while the high $-J$ lines are dominated by a hot, denser gas layer with $T_{\text {kin }}=500-1500 \mathrm{~K}$, and $n=(1-5) \times 10^{6} \mathrm{~cm}^{-3}$. The terminal bowshock consists of gas with moderate excitation, with a temperature in the range $T_{\text {kin }}=400-500 \mathrm{~K}$, and density $n=(1-$ 2) $\times 10^{6} \mathrm{~cm}^{-3}$. These observations show a complex excitation structure of the outflow, with the $\mathrm{CO}(5-4)$ line (close to what we later call mid- $J$ CO lines) contributing mostly to a relatively low-excitation component from the jet and cavity, and a moderate excitation component from the bowshock.

In a previous paper (Gómez-Ruiz et al. 2013, hereafter Paper I), we presented a study of the CO (6-5) and (7-6) emission (throughout the paper referred to as mid- $J$ CO transitions) in two low-mass class 0 outflows, namely L1448 and HH211. In both objects we found $\mathrm{CO}(6-5)$ and (7-6) emission tracing bipolar structures. In the case of L1448, extremely high-velocity emission (EHV), i.e. emission at relative velocities $>50 \mathrm{~km} \mathrm{~s}^{-1}$ with respect to the cloud velocity, was detected in both transitions. By performing a large velocity gradient (LVG) analysis, we inferred that the gas related with the outflow structures is dense $\left(>10^{5} \mathrm{~cm}^{-3}\right)$ and warm $(T>200 \mathrm{~K})$. It was determined that the EHV emission shows higher densities and temperatures than the high-velocity emission. Based on the LVG results and the position-velocity distribution of the emitting gas, we concluded that these mid- $J$ CO transitions are good tracers of molecular material likely related to the primary jet and to the highly excited bow-shock region in protostellar outflows.

In the present paper we extend the study of mid- $J$ CO emission to outflows from IM protostars in the Orion molecular cloud-2/3 (OMC-2/3), including Class 0- and I-type objects. The main purpose of this paper is to present the study of the distribution of the warm gas traced by the $\mathrm{CO}(6-5)$ and (7-6) transitions in IM outflows and compare it with the low-mass case. By incorporating lower- $J \mathrm{CO}$ transitions in our analysis, the excitation and physical conditions of IM outflows, as traced by these lines, were also studied.

\section{Target regions}

OMC-2/3 is located north of the Orion-A Giant Molecular Cloud (OMC 1). The distance to OMC 1 range from $\sim 390 \mathrm{pc}$ to $440 \mathrm{pc}$, given its 3-D structure determined by recent astrometry data (Kounkel et al. 2018). This region is one of the nearest clusterforming sites with IM protostars (e.g. Takahashi et al. 2006, 2008, here after T08). In this region, filamentary molecular clouds were discovered in the millimeter/submillimeter continuum observations with the IRAM-30m telescope and the JCMT (Chini et al. 1997; Johnstone \& Bally 1999). Within the filaments, millimeter/submillimeter continuum sources are found, presumably tracing prestellar and protostellar cores (e.g., Takahashi et al. 2009, 2012, 2013; Takahashi \& Ho 2012; Chini et al. 1997). Follow-up $\mathrm{CO}$ and $\mathrm{H}_{2}$ molecular line observations revealed the presence of a number of outflows originating from

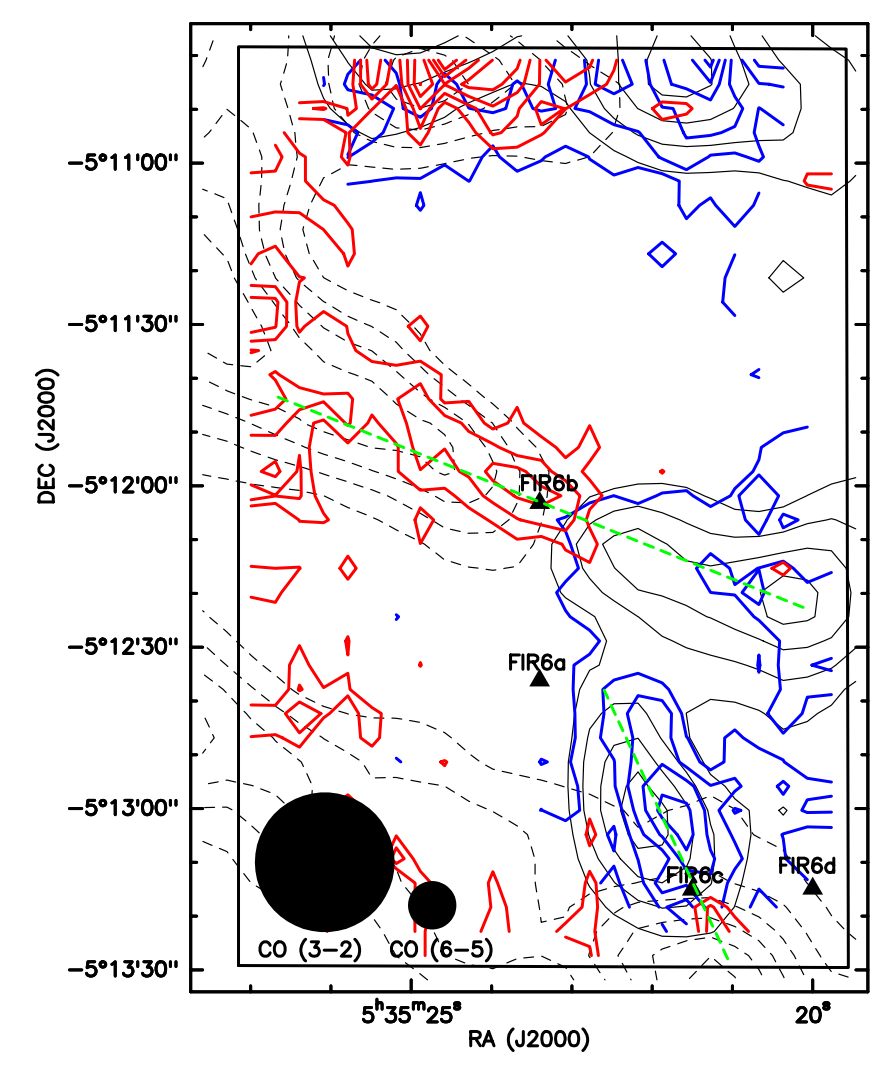

Fig. 1. CO (6-5) outflow emission in the OMC-2 FIR 6 region. Blue contours are for the blue-shifted emission in the range from -12 to $+8 \mathrm{~km} \mathrm{~s}^{-1}$; while red contours are for the red-shifted emission from +14 to $+30 \mathrm{~km} \mathrm{~s}^{-1}$. The first contour is $3 \sigma$, with contour spacing of $2 \sigma\left(\sigma=5.5\right.$ and $2.4 \mathrm{~K} \mathrm{~km} \mathrm{~s}^{-1}$, for blue- and red-shifted emission, respectively). The black solid and dashed contours are the blue- and red-shifted CO (3-2) emission, respectively, as presented by T08. The black square shows the whole field covered by our CHAMP+ observations. The triangles show the positions of the continuum sources and the green dashed lines indicate the outflow orientation (both according to T08).

some of the continuum sources (T08; Yu et al. 1997; Aso et al. 2000; Stanke et al. 2002; Williams et al. 2003). One of the most extensive molecular outflow surveys in the OMC-2/3 region was conducted by T08. By observing the CO (3-2) transition with the ASTE telescope, these authors detected 14 molecular outflows, indicative of a high ongoing star formation activity in this region.

From the CO (3-2) outflow survey of T08 and its follow-up observations, we selected the most promising sources in terms of outflow activity (bipolar morphology and high-velocity emission) and early evolutionary stage (class 0-type sources). Under these criteria, the regions around FIR (far-infrared) 6 in OMC-2 and MMS (millimeter source) 1-6 in OMC-3 (hereafter OMC-2 FIR 6 and OMC-3 MMS 1-6, respectively) were selected for follow-up observations in mid- $J$ CO lines. For these sources the derived luminosities are in the range of 6-251 and 9-150 $L_{\odot}$, respectively, which makes them very likely IM protostars (T08, Takahashi et al. 2009; Furlan et al. 2016). The envelope masses determined by T08 $\left(>3 M_{\odot}\right)$ are consistent with values of IM protostars.

OMC-2 FIR 6 contains at least four millimeter continuum sources named FIR 6a, 6b, 6c, and 6d (see Fig. 1). T08 reported the FIR 6b source as being a likely Class I-type object (based on their SED), while the lack of sufficient information for the 


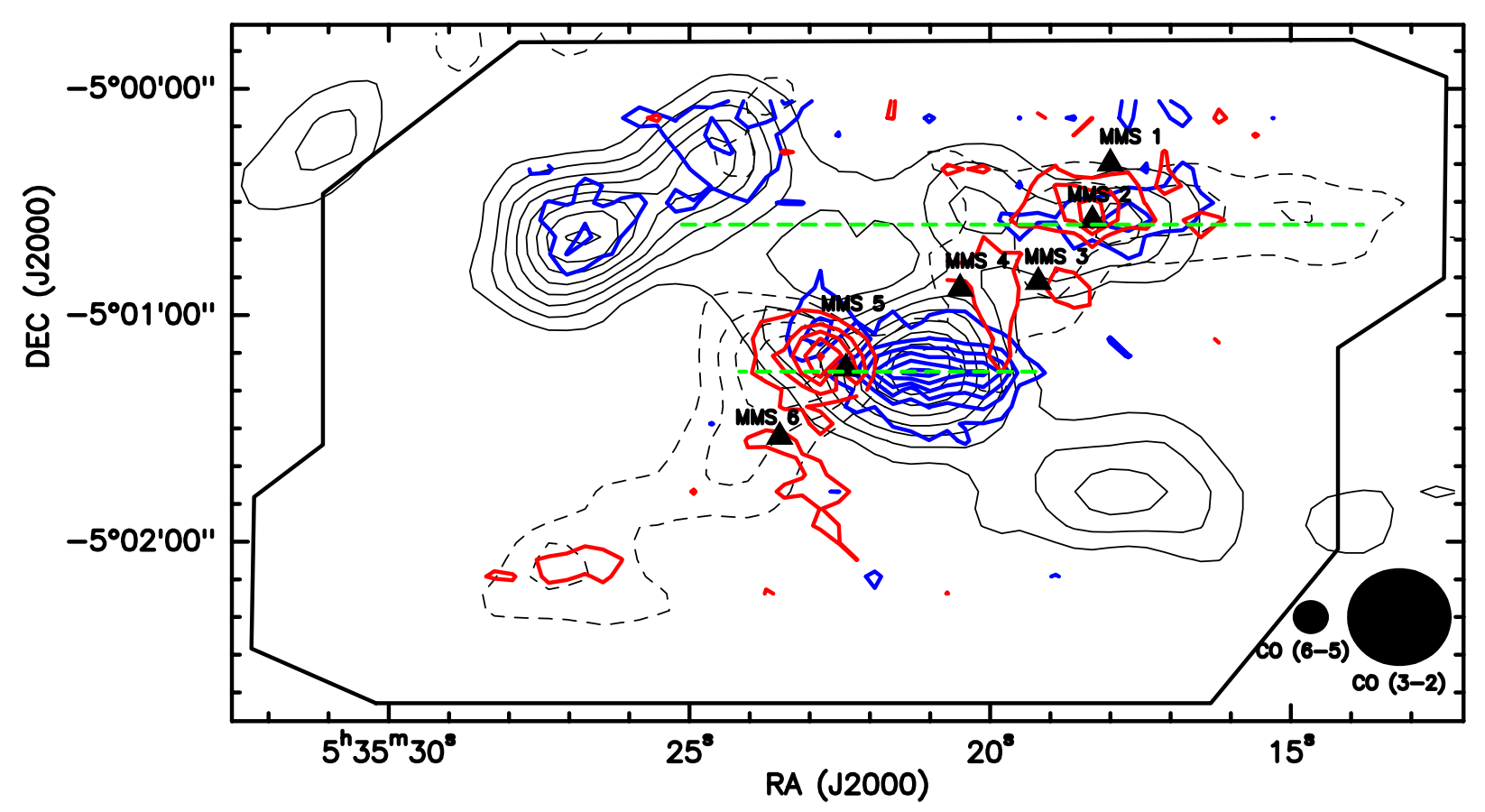

Fig. 2. CO (6-5) outflow emission in the OMC-3 MMS 1-6 region. Blue contours are for the blue-shifted emission in the range from -25 to $+7 \mathrm{~km} \mathrm{~s}^{-1}$; while red contours are for the red-shifted emission from +16 to $+31 \mathrm{~km} \mathrm{~s}^{-1}$. The first contour is $3 \sigma$ and contour spacing is $2 \sigma(\sigma=1.9$ and $0.8 \mathrm{~K} \mathrm{~km} \mathrm{~s}^{-1}$, for blue- and red-shifted emission, respectively). The black solid and dashed contours are the blue- and red-shifted CO (3-2) emission, respectively, as presented by T08. The black square shows the whole field covered by our CHAMP+ observations. The triangles show the positions of the continuum sources and the green dashed lines indicate the outflow orientation (both according to T08).

rest of the sources prevents us from clearly assessing their evolutionary status. The $\mathrm{CO}(3-2)$ data towards OMC-2 FIR 6 region revealed that two molecular outflows are driven by FIR $6 \mathrm{~b}$ and 6c (see Fig. 1). As a follow-up study of T08, Shimajiri et al. (2009) observed the OMC-2 FIR 6 region with the Nobeyama Millimeter Array in the $3 \mathrm{~mm}$ continuum, $\mathrm{CO}(1-0)$, and $\mathrm{SiO}$ (2-1) transitions. The two outflows found in $\mathrm{CO}(3-2)$ were confirmed in $\mathrm{CO}(1-0)$, in addition to a third outflow emanating from FIR $6 \mathrm{~d}$. The $\mathrm{SiO}(2-1)$ emission was found only at the bluelobe of FIR $6 \mathrm{c}$ outflow, but no $\mathrm{SiO}$ emission was clearly detected towards the $6 \mathrm{~b}$ outflow. From the line ratio between the $\mathrm{CO}(3-2)$ and (1-0) transitions, it was found that kinetic temperature in the outflow lobes is $~ 50 \mathrm{~K}$ (T08; Shimajiri et al. 2009).

The OMC-3 MMS 1-6 region consists of six millimeter continuum sources, named by Chini et al. (1997) as MMS (see Fig. 2). A clear identification of the evolutionary status through the SED was given for MMS 2 and MMS 5, which are class Iand class 0-type, respectively (T08; Takahashi et al. 2009). The MMS 2 source is actually a binary system of class I sources (T08; Tsujimoto et al. 2004). T08 reported clear bipolar outflow morphologies originating from MMS 2 and MMS 5 in the CO (3-2) emission. In the case of MMS 6, subarcsec resolution observations with the SMA in the CO (3-2) line by Takahashi \& Ho (2012) revealed an extremely compact molecular outflow (lobe size of $\approx 800 \mathrm{AU}$ ) associated with the continuum peak named MMS 6-main. Furthermore, Takahashi et al. (2012) reported that MMS 6-main is most likely in the protostellar phase. No clear molecular outflow signatures are detected in the other MMS sources, so that they are likely in the prestellar phase (Takahashi et al. 2013).

\section{APEX observations}

In this section we describe the observations made with two instruments of the APEX observatory.

\subsection{CHAMP+ observations}

Submillimeter observations towards OMC-3 MMS 1-6 and OMC-2 FIR 6 were performed with the MPIfR principal investigator (PI) instrument $\mathrm{CHAMP}^{+}$(The Carbon Heterodyne Array of the MPIfR, Kasemann et al. 2006) on the APEX telescope (Atacama Pathfinder EXperiment, Güsten et al. 2006) during November 2008 and July 2010, respectively. CHAMP ${ }^{+}$is a dualcolor $2 \times 7$ pixel heterodyne array for operation in the $450 \mu \mathrm{m}$ (low-frequency array, LFA) and $350 \mu \mathrm{m}$ (high-frequency array, HFA) atmospheric windows. The optics allow simultaneous observations in both colors. Both subarrays present a hexagonal arrangement. The front-end was connected to a fast Fourier transform spectrometer (FFTS, Klein et al. 2006).

The CO (6-5) and (7-6) lines were mapped simultaneously in the on-the-fly (OTF) mode, with an ON time of 0.5 second per position, with steps of $3^{\prime \prime}$. For the OMC-2 FIR 6 region a map of $60^{\prime \prime} \times 120^{\prime \prime}$ was centered at the position of the FIR $6 \mathrm{~b}$ source. For the OMC-3 MMS 1-6 region a map with of $280^{\prime \prime} \times 150^{\prime \prime}$ was centered at the position of MMS 5. Table 1 shows the coordinates (right ascension and declination) of the central position of the maps and their average rms. Additional single-pointing observations were done on two selected positions of OMC-2 FIR 6 (called FIR 6b and FIR 6c-B1; see Sect. 4) in a setup consisting of ${ }^{13} \mathrm{CO}(6-5)$ and $\mathrm{HCO}^{+}(9-8)$. No emission was detected in the $\mathrm{HCO}^{+}(9-8)$ line, with upper limits of $186 \mathrm{mK}\left(T_{\mathrm{A}}^{*}\right)$, and therefore was not further discussed. For all observations, the backend was set to provide a total bandwidth of $2.8 \mathrm{GHz}$ divided in 8192 channels for each pixel. The final spectra were re-sampled to $1.0 \mathrm{~km} \mathrm{~s}^{-1}$ spectral resolution for all transitions in both sources.

During the observations, the precipitable water vapor (PWV) was in the range of $0.3-0.7 \mathrm{~mm}$. The pointing was determined by CO (6-5) cross-scan observations on IK Tau. Pointing accuracy was always within $3^{\prime \prime}$. The focus was checked on Jupiter and Mars. The calibration was done by observing hot and cold 
Table 1. Map central position and rms.

\begin{tabular}{lccccc}
\hline \hline & & & \multicolumn{3}{c}{ rms. $(\mathrm{K}){ }^{(a)}$} \\
\cline { 4 - 7 } Source & $\alpha(\mathrm{J} 2000)$ & $\delta(\mathrm{J} 2000)$ & \multicolumn{2}{c}{${ }^{12} \mathrm{CO}$} & $\mathrm{SiO}$ \\
& $\left(\mathrm{(} \mathrm{m} \mathrm{s}^{2}\right)$ & $\left({ }^{\circ} \prime^{\prime \prime}\right)$ & $(6-5)$ & $(7-6)$ & $(5-4)$ \\
\hline OMC-2 FIR 6b & $05: 35: 23.4$ & $-05: 12: 03$ & 0.7 & 3.0 & 0.05 \\
OMC-3 MMS 5 & $05: 35: 22.5$ & $-05: 01: 15$ & 0.5 & 1.9 & - \\
\hline
\end{tabular}

Notes. ${ }^{(a)}$ Average rms $\left(T_{\mathrm{MB}}\right)$ in a channel map of $1.0 \mathrm{~km} \mathrm{~s}^{-1}$.

Table 2. Characteristics of the observed transitions.

\begin{tabular}{lcccc}
\hline \hline Line & $\begin{array}{c}v_{0} \\
\mathrm{GHz}\end{array}$ & $\begin{array}{c}A \\
10^{-5} \mathrm{~s}^{-1}\end{array}$ & $\begin{array}{c}E_{\mathrm{u}} / k \\
\mathrm{~K}\end{array}$ & $\begin{array}{c}H P B W \\
{ }^{\prime}\end{array}$ \\
\hline $\mathrm{CO}(6-5)$ & 691.473 & 2.137 & 116.16 & 9.0 \\
$\mathrm{CO}(7-6)$ & 806.651 & 3.422 & 154.87 & 7.7 \\
${ }^{13} \mathrm{CO}(6-5)$ & 661.067 & 299.0 & 111.05 & 9.4 \\
$\mathrm{SiO}(5-4)$ & 217.107 & 51.96 & 31.26 & 30.5 \\
$\mathrm{HCO}^{+}(9-8)$ & 802.458 & 4400 & 192.58 & 7.8 \\
\hline
\end{tabular}

Notes. Molecular data taken from LAMDA (Schöier et al. 2005).

loads. In our observations the OFF position was not checked for emission, but this position was selected to avoid regions known to present emission in low $-J \mathrm{CO}$ transitions. However, due to an erroneous selection of the OFF position during the ${ }^{13} \mathrm{CO}(6-5)$ and $\mathrm{HCO}^{+}(9-8)$ single-pointing observations of the FIR $6 \mathrm{~b}$ position, contamination of the OFF position was found in these spectra. Standard data reduction procedures such as flagging bad spectra and base line subtraction were done with CLASS, while images were produced with the XY_MAP task in GReG, both programs being part of the GILDAS software ${ }^{1}$. The griding procedure in XY_map was made to provide a final resolution equal to the half power beamwidth (HPBW) of the telescope at the observed frequency. Table 2 lists the observed transitions and rest frequencies $\left(v_{0}\right)$, their corresponding HPBW, upper state energy $\left(E_{\mathrm{u}}\right)$, and Einstein coefficient $(A)$.

The images and spectra are presented in main beam brightness temperature scale, $T_{\mathrm{MB}}=T_{\mathrm{A}}^{*} / \eta_{\mathrm{MB}}\left(\eta_{\mathrm{MB}}=\eta_{\mathrm{s}} / F_{\text {eff }}\right)$, for which we have used a forward efficiency $\left(F_{\text {eff }}\right)$ of 0.95 and a beam coupling efficiency $\left(\eta_{\mathrm{s}}\right)$ measured on Jupiter. During the OMC-2 observations the measured $\eta_{\mathrm{s}}$ was 0.48 for both LFA and HFA, while during the OMC-3 observations $\eta_{\mathrm{s}}$ was 0.48 and 0.45 for LFA and HFA, respectively. Based on the variation of the integrated mid- $J \mathrm{CO}$ flux observed in the line pointing sources, we estimated a $20 \%$ calibration uncertainty for both LFA and HFA (see also Yildız et al. 2015).

\subsection{APEX-1 observations}

Complementary mapping observations in the $\mathrm{SiO}$ (5-4) line were carried out towards OMC-2 FIR 6 with the APEX-1 facility instrument in September 2010. The receiver was connected to a FFTS that provides a bandwidth of $1 \mathrm{GHz}$ and 4096 channels, which at the rest frequency of $\mathrm{SiO}(5-4)$ resulted in a spectral resolution of $0.3 \mathrm{~km} \mathrm{~s}^{-1}$. The OTF map with a size of $70^{\prime \prime} \times 70^{\prime \prime}$, in steps of $9^{\prime \prime}$, was centered at the position of the FIR $6 \mathrm{~b}$ source. Therefore, the map covers the central parts of the FIR $6 \mathrm{~b}$

\footnotetext{
1 http://iram.fr/IRAMFR/PDB/gildas/gildas.html
}

Table 3. Coordinates of the outflow peak positions.

\begin{tabular}{lccc}
\hline \hline Position & $\begin{array}{c}\alpha(\mathrm{J} 2000) \\
(\mathrm{h} \mathrm{m} \mathrm{s})\end{array}$ & $\begin{array}{c}\delta(\mathrm{J} 2000) \\
\left({ }^{\circ}{ }^{\prime \prime}\right)\end{array}$ & $\begin{array}{c}v_{\min }, v_{\max }{ }^{(*)} \\
\left(\mathrm{km} \mathrm{s}^{-1}\right)\end{array}$ \\
\hline FIR 6b-B1 & $05: 35: 21.2$ & $-05: 12: 15$ & 5,8 \\
FIR 6b-R1 & $05: 35: 23.7$ & $-05: 11: 59$ & 14,30 \\
FIR 6c-B1 & $05: 35: 21.6$ & $-05: 13: 00$ & $-16,8$ \\
FIR 6c-B2 & $05: 35: 21.7$ & $-05: 13: 00$ & $-10,8$ \\
MMS 5-B1 & $05: 35: 21.3$ & $-05: 01: 16$ & $-95,7$ \\
MMS 5-R1 & $05: 35: 22.5$ & $-05: 01: 14$ & 16,75 \\
MMS 2-B1 & $05: 35: 18.2$ & $-05: 00: 32$ & $-12,7$ \\
MMS 2-R1 & $05: 35: 17.7$ & $-05: 00: 34$ & 16,30 \\
\hline
\end{tabular}

Notes. ${ }^{(*)}$ Velocity boundaries of the outflow emission (see Sect. 4).

outflow lobes and the blue lobe of the FIR $6 \mathrm{c}$ outflow. The pointing accuracy was checked by $\mathrm{CO}(2-1)$ cross-scan observations on IK Tau. Pointing accuracy was always within $5^{\prime \prime}$. The focus was checked on Jupiter and Mars. The calibration was done by observing hot and cold loads. The data reduction and image processing are similar to the procedure described for the CHAMP+ observations, using CLASS and GReG. The final spectra were re-sampled to a $2.0 \mathrm{~km} \mathrm{~s}^{-1}$ spectral resolution. In order to convert to $T_{\mathrm{MB}}$ scale, the nominal values of 0.95 and 0.75 were adopted for the forward and beam coupling efficiency, respectively. A calibration uncertainly of $20 \%$ is also expected for this receiver, based on the flux variation of the line pointing sources.

\section{Results}

In order to define the outflow velocity range we use the $\mathrm{CO}(6-5)$ channel maps and the spectra taken at different outflow positions. We defined the lower boundary limit $\left(v_{\min }\right)$ by looking for outflow morphologies clearly separated from the ambient diffuse emission. We then established $v_{\max }$ from the spectra taken at the peak positions within the outflow's lobes. In our case, $v_{\max }$ was defined as the velocity in which the emission in the spectrum drops below the $3 \sigma$ level. The velocity limits for all identified outflows and the positions of the outflow peaks are given in Table 3. Based on the measurements of the $\mathrm{H}^{13} \mathrm{CO}^{+}(1-0)$ line by Aso et al. (2000), throughout the paper we assume the cloud LSR velocity $\left(V_{\mathrm{LSR}}\right)$ of $+11 \mathrm{~km} \mathrm{~s}^{-1}$.

\subsection{OMC-2 FIR 6 outflows}

In the following we describe the results obtained for the $\mathrm{CO}$ (6-5) and $\mathrm{CO}(7-6),{ }^{13} \mathrm{CO}^{+}(6-5)$, and $\mathrm{SiO}(5-4)$, respectively.

\subsection{1. $\mathrm{CO}(6-5)$ and $\mathrm{CO}(7-6)$ emission}

Figure 1 shows the $\mathrm{CO}(6-5)$ emission detected in the FIR 6 region, integrated along the blue- and red-shifted wings. Figure 1 also shows the $\mathrm{CO}(3-2)$ wing emission from T08. We found the two outflows related to FIR $6 \mathrm{~b}$ and FIR $6 \mathrm{c}$, reported previously in $\mathrm{CO}$ (3-2) by T08. Both outflow lobes from FIR $6 \mathrm{~b}$ are detected in $\mathrm{CO}(6-5)$, and also the blue-shifted lobe from FIR $6 \mathrm{c}$ (the redshifted lobe was not covered by our map). Four emission peaks were found at the outflow positions, which we list in Table 3 . The $\mathrm{CO}(6-5)$ emission from FIR6b seems to peak closer to the central source than the $\mathrm{CO}(3-2)$ emission. Convolutions to the $\mathrm{CO}$ (3-2) beam confirms that this tendency is not an effect of the different angular resolutions. On the other hand, the peak position 


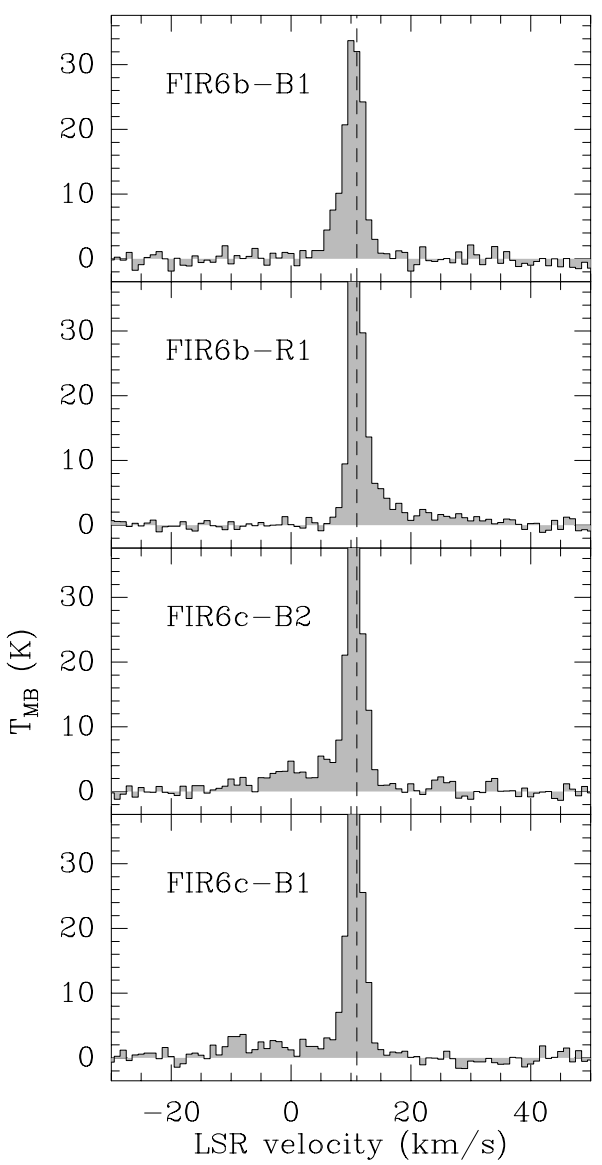

Fig. 3. CO (6-5) sample spectra at selected positions (see Table 3) within FIR 6 outflows $\left(1 \mathrm{~km} \mathrm{~s}^{-1}\right.$ spectral resolution). The dashed line indicates the cloud velocity.

of the CO (6-5) emission in the blue lobe of FIR $6 \mathrm{c}$ outflow is consistent with the location of the CO (3-2) emission peak. No high-velocity emission tracing outflows from FIR 6a and FIR 6d was found. In the case of FIR $6 \mathrm{~d}$ we are not able to unambiguously probe the outflow emission, since the outflow structures revealed by previous low $-J \mathrm{CO}$ observations are located right at the edge of our map. Around the northern edge of the map, blueand red-shifted emission is also noticed; however, these structures are not classified as a clear outflow by T08, and therefore we exclude these structures in the analysis that follows.

Figure 3 shows the spectra taken at the position of the emission peak of each lobe. In all positions, the peak of the $\mathrm{CO}(6-5)$ profiles is found at the cloud systemic velocity $\left(V_{\mathrm{LSR}} \approx 11.0 \mathrm{~km} \mathrm{~s}^{-1}\right)$. Three positions show wings (FIR 6b-R1) and secondary peaks at high velocities (FIR 6c-B1 and FIR 6c$\mathrm{B} 2)$. At the FIR $6 \mathrm{~b}-\mathrm{B} 1$ position the line profile only extends down to $V_{\mathrm{LSR}}=5 \mathrm{~km} \mathrm{~s}^{-1}$.

The CO (7-6) emission was detected mostly at low velocities $\left(V_{\mathrm{LSR}}=8-14 \mathrm{~km} \mathrm{~s}^{-1}\right)$, tracing the cloud and/or envelope emission. At the outflow peaks the $\mathrm{CO}(7-6)$ emission is barely noticed $(S / N<5)$ in the spectra.

\subsection{2. ${ }^{13} \mathrm{CO}(6-5)$ emission and opacity estimation}

In Fig. 4 we show the ${ }^{13} \mathrm{CO}(6-5)$ spectra taken at two positions within the FIR 6 region: the FIR 6b source and FIR 6c-B1. The ${ }^{13} \mathrm{CO}(6-5)$ emission is only detected in about three channels, around the cloud velocity. All spectra in Fig. 4 show the emission peaking at the systemic velocity $\left(V_{\mathrm{LSR}}=11 \mathrm{~km} \mathrm{~s}^{-1}\right)$, with a FWHM of $\sim 2 \mathrm{~km} \mathrm{~s}^{-1}$. At the FIR $6 \mathrm{~b}$ position an absorption feature is also present at $V_{\mathrm{LSR}}=9 \mathrm{~km} \mathrm{~s}^{-1}$, which is more evident in the external pixels. This absorption feature is likely due to contamination from the OFF position (see Sect. 2), and thus the ${ }^{13} \mathrm{CO}(6-5)$ emission detected at the FIR $6 \mathrm{~b}$ position should be taken as a lower limit.

Based on the ${ }^{13} \mathrm{CO}(6-5)$ observations, we estimate the ${ }^{12} \mathrm{CO}$ (6-5) line opacity at the FIR $6 \mathrm{~b}$ and FIR $6 \mathrm{c}-\mathrm{B} 1$ positions. We follow the formulation introduced in Paper I, and use the assumption that the ${ }^{13} \mathrm{CO}$ emission is optically thin and that ${ }^{12} \mathrm{CO}$ and ${ }^{13} \mathrm{CO}$ have the same excitation temperature, which means that the ${ }^{12} \mathrm{CO}$ to ${ }^{13} \mathrm{CO}$ antenna temperature line ratio provide a measure of the ${ }^{12} \mathrm{CO}$ opacity, $\tau_{12}$ (see Eq. (1) of Paper I). Our opacity calculations were made assuming a $\left[{ }^{12} \mathrm{CO}\right] /\left[{ }^{13} \mathrm{CO}\right]$ abundance ratio of 50 (Kahane et al. 2018). We also note that the error of the ratios does not require additional calibration uncertainties, since the observations were made with the same instrument and within the same band. In Table 4 we show the opacities estimated for the FIR6b and FIR6c-B1 positions. These estimations are performed using the three channels around the cloud velocity. The $\tau_{12}$ values vary from $\sim 2$ to 10 . Therefore we see that the ${ }^{12} \mathrm{CO}$ is optically thick at the cloud velocity. On the other hand, in the outflow velocity range, where ${ }^{13} \mathrm{CO}$ was not detected, the $3 \sigma$ upper limits indicate that the emission can be optically thin: with an intensity ratio $>50, \tau_{12}$ is $<0.4$. Based on these results, in the calculations presented in the analysis, to determine the gas excitation and outflow properties, we assume that the ${ }^{12} \mathrm{CO}(6-5)$ high-velocity emission is optically thin in the FIR 6 outflows, and in this way use the simple formulations for the optically thin case. Although we did not observe the ${ }^{13} \mathrm{CO}(6-5)$ line in MMS $1-6$ region, we also assume that the ${ }^{12} \mathrm{CO}(6-5)$ high-velocity emission from the outflows in that region is optically thin.

\subsection{3. $\mathrm{SiO}(5-4)$ emission}

The SiO (5-4) emission is only detected to the SW of FIR $6 b$, in the velocity range from -12 to $15 \mathrm{~km} \mathrm{~s}^{-1}$. The upper panel of Fig. 5 shows the $\mathrm{SiO}(5-4)$ integrated emission map. The $\mathrm{SiO}$ emission is located towards the region that corresponds to the blue-lobe of the FIR $6 \mathrm{c}$ outflow. The angular resolution $\left(\sim 30^{\prime \prime}\right)$ of the $\mathrm{SiO}(5-4)$ observations is not sufficient to allow a clear identification of outflow structures. The lower panel of Fig. 5 shows the $\mathrm{SiO}(5-4)$ spectrum averaged over the area in which the emission is detected (indicated by the green polygon in the upper panel of Fig. 5). The $\mathrm{SiO}$ (5-4) averaged spectrum shows a velocity range $-12 \leq V_{\mathrm{LSR}} \leq+15 \mathrm{~km} \mathrm{~s}^{-1}$. The lower panel of Fig. 5 also shows a comparison with the CO (6-5) spectrum averaged over the same region, obtained from a map convolved to the same angular resolution. The velocity range is similar, suggesting that the $\mathrm{SiO}(5-4)$ and $\mathrm{CO}(6-5)$ are tracing the same outflow gas. However, while the $\mathrm{CO}$ profile shows a typical winglike profile with a main peak at low velocities, the $\mathrm{SiO}$ is not wing-like and with a secondary peak at high velocities $\left(V_{\mathrm{LSR}} \sim\right.$ $\left.-8 \mathrm{~km} \mathrm{~s}^{-1}\right)$. This behavior, also observed in other outflows, is indicative of the uniqueness of the $\mathrm{SiO}$ as a shock tracer (e.g., L1448: Paper I; Bachiller et al. 1990). In CO (6-5) an indication of secondary peaks at high velocities is only observed in the spectra at the original angular resolution (Fig. 3), in fact at about the same $\mathrm{SiO}(5-4)$ velocity range.

Our APEX SiO (5-4) data showed a $\mathrm{SiO}$ gas distribution similar to that found in previous interferometric $\mathrm{SiO}$ (2-1) observations by Shimajiri et al. (2009). In both cases the $\mathrm{SiO}$ emission was detected only towards the region that corresponds to the blue 

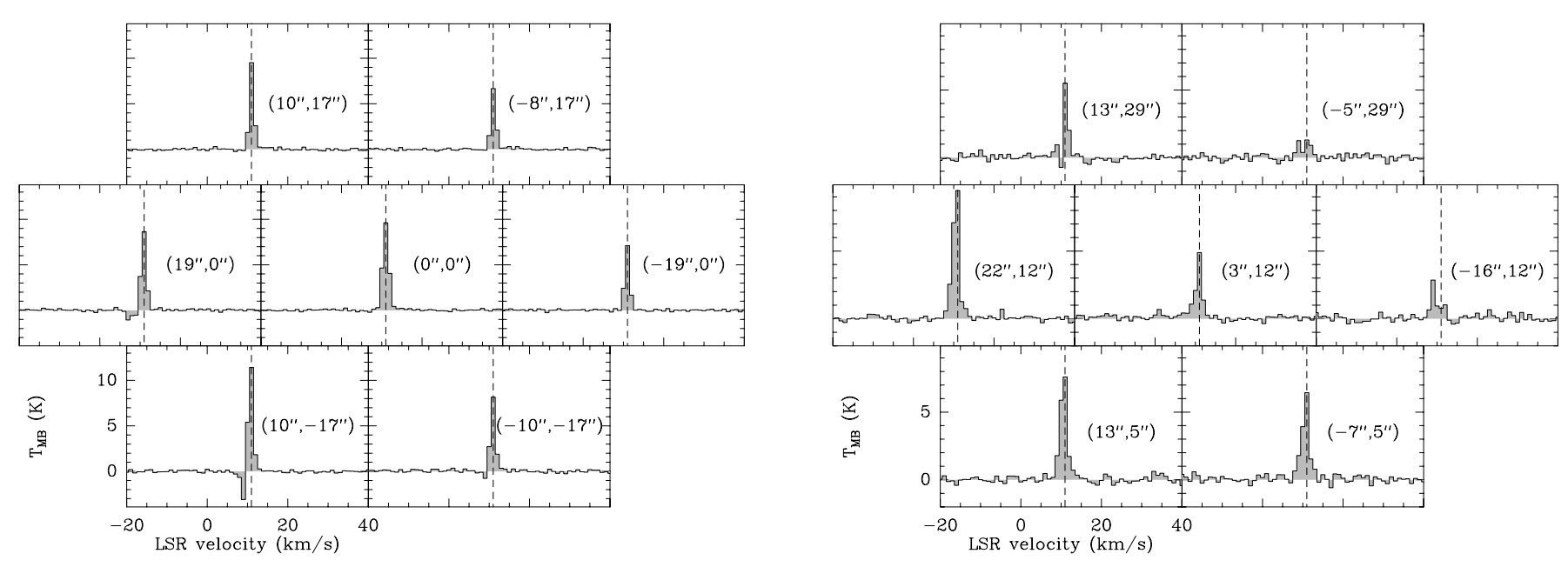

Fig. 4. ${ }^{13} \mathrm{CO}(6-5)$ spectra taken at selected positions within FIR 6 region $\left(1 \mathrm{~km} \mathrm{~s}^{-1}\right.$ spectral resolution). The figure shows the seven CHAMP+ pixels for each pointing observation. Left: observation centered on FIR 6b. Right: observation centered on FIR 6c-B1 position (offsets with respect to FIR 6c).

Table 4. ${ }^{12} \mathrm{CO}(6-5)$ opacity around cloud $V_{\mathrm{LSR}}$ in FIR $6 \mathrm{~b}$ and FIR 6c-B1.

\begin{tabular}{lcc}
\hline \hline $\begin{array}{l}V_{\mathrm{LSR}} \\
\left(\mathrm{km} \mathrm{s}^{-1}\right)\end{array}$ & $\begin{array}{c}{ }^{12} \mathrm{CO} /{ }^{13} \mathrm{CO}(6-5) \\
T_{\mathrm{MB}} \text { ratio }\end{array}$ & Opacities \\
\hline \multicolumn{3}{c}{ FIR 6b } \\
\hline 10 & $8.6(0.3)$ & $5.8(0.3)$ \\
11 & $4.8(0.1)$ & $10.4(0.2)$ \\
12 & $7.3(0.3)$ & $6.8(0.3)$ \\
\hline & FIR 6c-B1 \\
\hline 10 & $10.1(0.5)$ & $4.9(0.2)$ \\
12 & $5.5(0.2)$ & $9.1(0.3)$ \\
\hline
\end{tabular}

Notes. Parenthesis show statistical errors.

lobe of the FIR $6 \mathrm{c}$ outflow. Our larger map confirms that no $\mathrm{SiO}$ emission is clearly detected from the FIR $6 \mathrm{~b}$ outflow. Similar cases where $\mathrm{CO}$ outflows do not present an $\mathrm{SiO}$ counterpart have been reported in the literature (see Zapata et al. 2006, for discussion).

\subsection{OMC-3 MMS 1-6 outflows: $C O$ (6-5) and CO (7-6) emission}

Figure 2 shows the region covered by our $\mathrm{CO}(6-5)$ and (7-6) maps. We clearly identified the CO (6-5) outflow emission originating from the sources MMS 5 and MMS 2. We identified four outflow peaks, listed in Table 3. These two outflows were previously reported by $\mathrm{T} 08$. However the compact outflow detected in CO (3-2) by Takahashi \& Ho (2012) emanating from MMS 6 was not detected in $\mathrm{CO}(6-5)$. The nondetection of this outflow is possibly due to beam dilution, since even with Nobeyama interferometric CO (1-0) observations by Takahashi et al. (2009), with an angular resolution of several arcseconds, the compact outflow was not detected (see also discussion by Takahashi \& Ho 2012). No clear evidence of outflow activity towards the remaining MMS objects was found. The outflow emanating from MMS 5 seems to be orientated along the east-west direction, while for the outflow from MMS 2 the observations cannot clearly disentangle a tendency in the outflow direction. We also notice two blobs of blue-shifted emission on the far side of the putative MMS 2 blue-shifted outflow lobe, which coincide with similar structures detected in CO (3-2). Since T08 and Williams et al. (2003) could not unambiguously relate these structures with MMS2 (or the other nearby millimeter sources), for consistency with the later physical parameters comparisons, we did not take these structures into account in the following calculations. The most intense CO (6-5) emission is related to the MMS 5 outflow, which shows an extended blue lobe, while the red lobe shows a more compact structure.

A remarkable result from our $\mathrm{CO}(6-5)$ observations is the detection of EHV emission from the MMS 5 outflow. The EHV range is defined as $V_{\mathrm{LSR}}=-95$ to $-80 \mathrm{~km} \mathrm{~s}^{-1}$ for the blue-shifted lobe and $V_{\mathrm{LSR}}=60-70 \mathrm{~km} \mathrm{~s}^{-1}$ for the red-shifted lobe. In Fig. 6 we show the velocity integrated emission in the EHV range, overlaid on the near-infrared (NIR) emission ( $K$-band, $2.12 \mu \mathrm{m}$, taken from T08), and the spectra taken at the position of the peak intensity of the EHV structures. The integrated intensity maps show that the EHV emission is located within a radius of $\sim 10^{\prime \prime}$ from MMS 5. The blue-shifted EHV emission coincides with the inner collimated NIR structure. The CO (6-5) spectra clearly show secondary peaks related to these EHV features, with the red-shifted EHV component stronger than the blue-shifted one. The compact morphology and the characteristic line profiles suggest that these components are the equivalent of the so-called EHV bullets found in class 0 low-mass outflows (e.g. L1448mm: Bachiller et al. 1990, Paper I). Although evidence for EHV emission was not found in the CO (3-2) spectra observed by T08, recent ALMA observations of CO (2-1) and $\mathrm{SiO}(5-4)$ have revealed the EHV emission from MMS 5 (Matsushita et al. 2019).

The CO (7-6) high-velocity emission was detected in the outflows associated with MMS 5 and MMS 2 (see Fig. 7). The outflow lobes from MMS 5 in the CO (7-6) emission show a similar structure to those traced in the $\mathrm{CO}(6-5)$ emission. An EHV component in the CO (7-6) line is barely noticed at $3 \sigma$ only at the R1 position. In the case of the MMS 2 outflow, the $\mathrm{CO}(7-6)$ high-velocity emission is coming mainly from the redshifted lobe, with the blue-shifted lobe only detected at the $4 \sigma$ level. 

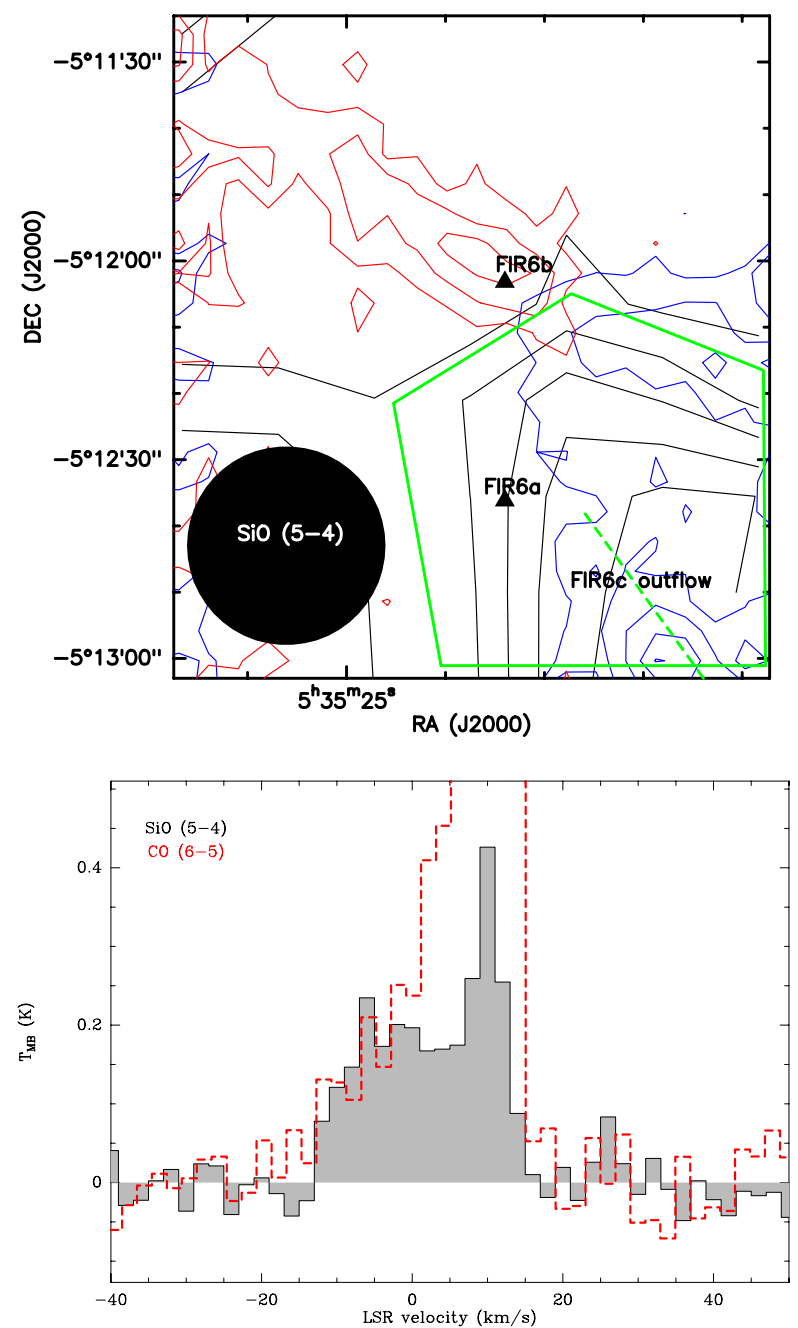

Fig. 5. Upper panel: $\mathrm{SiO}(5-4)$ map of the FIR 6 region, integrated from -12 to $15 \mathrm{~km} \mathrm{~s}^{-1}$ (black contours), overlaid with the CO (6-5) outflow emission (blue and red contours; as in Fig. 1). The $\mathrm{SiO}$ contour step is $3 \sigma$, with the first contour at $3 \sigma\left(\sigma=0.38 \mathrm{~K} \mathrm{~km} \mathrm{~s}^{-1}\right)$. Lower panel: $\mathrm{SiO}(5-4)$ spectrum (black) averaged over the region indicated by the green polygon on the top panel. To increase the signal-to-noise ratio the spectral resolution was smoothed to $2 \mathrm{~km} \mathrm{~s}^{-1}$. As comparison the $\mathrm{CO}$ (6-5) spectrum is shown in red.

\section{Analysis}

I the following we present the results of a radatiave transfer analysis using two methods, as well as the determination of the outflow parameters.

\subsection{Population diagram}

Under the assumption that the gas is in LTE and that the ${ }^{12} \mathrm{CO}$ emission is optically thin, we use the Boltzmann relation for the level populations to estimate the rotational temperature $\left(T_{\text {rot }}\right)$ and the total column density $(N)$ at selected positions within the outflow lobes, following the formulation presented in Paper I. The calculations were made for the emission from the outflow peaks reported in Table 3 (see also Figs. 1 and 2). The CO (3-2) data taken with ASTE by T08 were added to our APEX data. We convolved our $\mathrm{CO}(6-5)$ and (7-6) observations to the angular resolution of the $\mathrm{CO}(3-2)$ observations (i.e., 26"). Table 5 shows the CO (3-2, 6-5, 7-6) integrated intensities of the selected positions, convolved to $26^{\prime \prime}$ resolution. The emission is integrated between the outflow's wing limits, $v_{\min }$ and $v_{\max }$, which we assume to be optically thin (see Sect. 4). In the calculation we included the calibration uncertainties of each line, whose value is $20 \%$ for all the cases (including the ASTE data; see T08). For future references, we report separately in Table 5 the intensities for the EHV range, although we do not provide calculations for this component, given that the $\mathrm{CO}(3-2)$ and $\mathrm{CO}$ (7-6) lines were moslty not detected.

Table 6 shows the results, $T_{\text {rot }}$ and $N$, obtained from the rotational diagram analysis. To within the errors, most of the positions present a rotational temperature in the range of 60$70 \mathrm{~K}$, with the exception of the FIR 6b-B 1 position, which shows a lower temperature of $\sim 30 \mathrm{~K}$. In terms of the column density, higher values are found in the OMC-2 FIR 6 positions.

\subsection{Large velocity gradient}

Radiative transfer calculations with RADEX (van der Tak et al. 2007) were made in the LVG approximation and plane-parallel geometry. The molecular data was retrieved from the LAMDA database $^{2}$ (Schöier et al. 2005) and the collisional rate coefficients were adopted from Yang et al. (2010).

The RADEX calculations were used to reproduce simultaneously the observed $\mathrm{CO}(3-2) / \mathrm{CO}(6-5), \mathrm{CO}(3-2) / \mathrm{CO}(7-6)$, and $\mathrm{CO}(6-5) / \mathrm{CO}(7-6)$ integrated intensity ratios at different positions within the outflow lobes. The intensities are from Table 5, and we added the calibration uncertainties already reported; we note, however, that the ratios with the CHAMP+ lines themselves do not require additional calibration errors since they were observed simultaneously.

In Fig. 8, kinetic temperature ( $\left.T_{\text {kin }}\right)$ vs. $\mathrm{H}_{2}$ density $(n)$ plots are shown for each outflow position. In most of the cases only lower limits to kinetic temperature and $\mathrm{H}_{2}$ density can be provided, with values of $T_{\text {kin }}>30-50 \mathrm{~K}$ and $n>10^{4} \mathrm{~cm}^{-3}$. Only in the case of MMS 5-R1 the three ratios are able to set more stringent constraints to the density and a higher kinetic temperature lower limit: $n \approx 10^{4}-10^{5} \mathrm{~cm}^{-3}, T_{\text {kin }}>200 \mathrm{~K}$. These values indicate that the outflow driven by MMS 5 present physical conditions similar to those obtained in the low-mass class 0 outflows from L1448-mm and HH211 (see Paper I).

\subsection{Outflow parameters}

Using the velocity integrated $\mathrm{CO}(6-5)$ high-velocity maps (pixels with $\mathrm{S} / \mathrm{N}$ above 3 ), we calculate the $\mathrm{H}_{2}$ mass of the outflow gas, following the relations presented in Paper I between the total CO column density $(N)$ and the velocity integrated emission, under the assumption of optically thin ${ }^{12} \mathrm{CO}$ (shown to be the case, based on the opacity upper limit determined in Sect. 4 for the high-velocity gas at some outflow positions). The high-velocity maps are produced by integrating the emission between the minimun and maximum velocity of each outflow lobe, following the $v_{\min }, v_{\max }$ values reported in Table 3 . In the calculations we used the temperatures obtained from the rotational diagram analysis (Table 6), the standard ISM value for the relative ${ }^{12} \mathrm{CO}$ abundance, $\mathrm{X}\left(\mathrm{H}_{2} / \mathrm{CO}\right) \sim 10^{4}$ (e.g., Wilson \& Matteucci 1992), and a distance to the source of $400 \mathrm{pc}$ (which is close to the average of the distance range of Orion A 3D structure). As in Paper I, we point out that considering uncertainties such as data calibration, definition of the velocity boundary limits for the integrated emission, and opacity assumptions, the mass determinations are usually accurate within a factor of 2-3 (see, e.g., Cabrit \& Bertout 1990).

2 http://www.strw.leidenuniv.nl/ moldata/ 


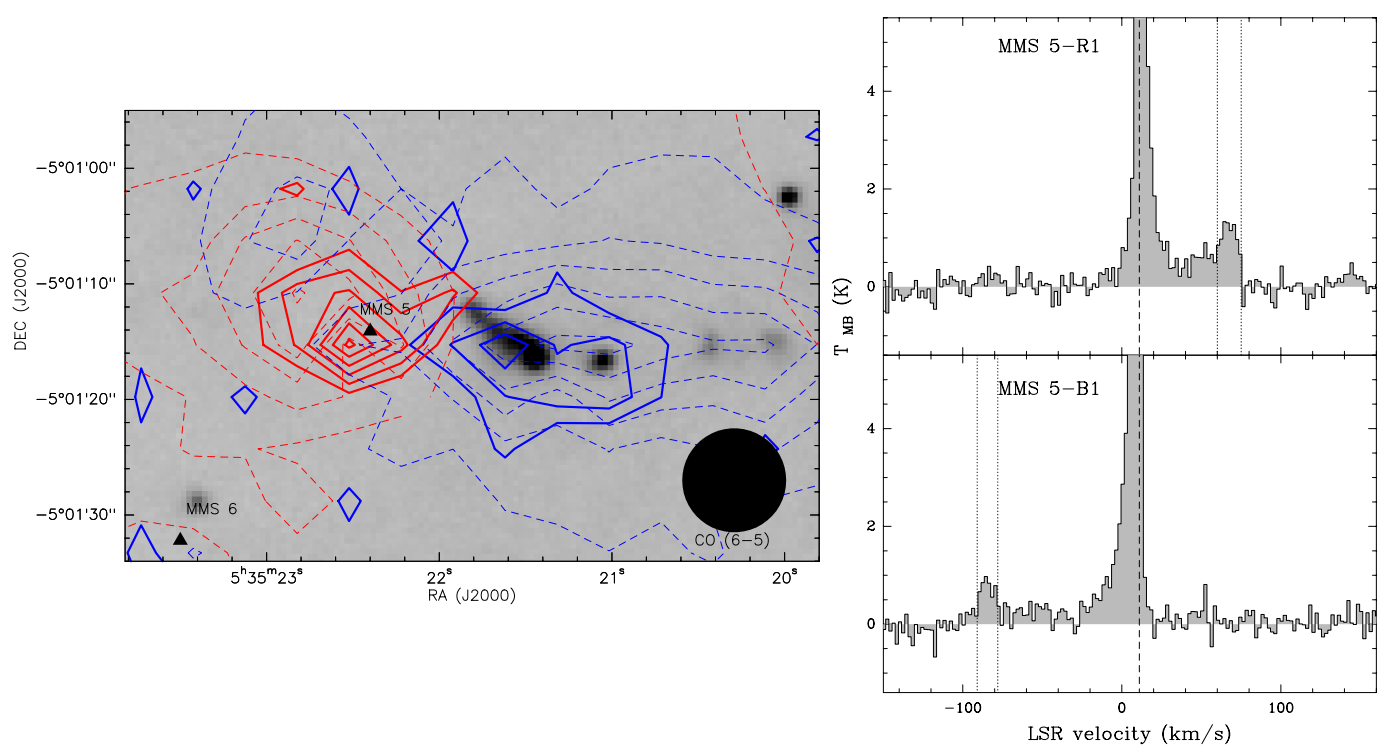

Fig. 6. Left: EHV CO (6-5) emission from MMS 5 outflow. The solid blue contours show the CO (6-5) emission integrated from $V_{\mathrm{LSR}}=-91$ to $-78 \mathrm{~km} \mathrm{~s}^{-1}$ (blue-shifted EHV range), while the solid red contours show the CO (6-5) emission integrated from $V_{\mathrm{LSR}}=60-75 \mathrm{~km} \mathrm{~s}^{-1}$ (red-shifted EHV range). The first contour is $3 \sigma$, with contour spacing in steps of $\sigma\left(2.2 \mathrm{~K} \mathrm{~km} \mathrm{~s}^{-1}\right)$. The dashed contours show the high-velocity CO (6-5) emission as presented in Fig. 2. The background greyscale image is the NIR ( $K$-band, $2.12 \mu \mathrm{m})$ map taken from T08. Right: CO (6-5) spectrum taken at the B1 and R1 positions (see Table 3). The vertical dot-dashed lines show the boundaries of the EHV range, while the dashed line indicates the cloud velocity.

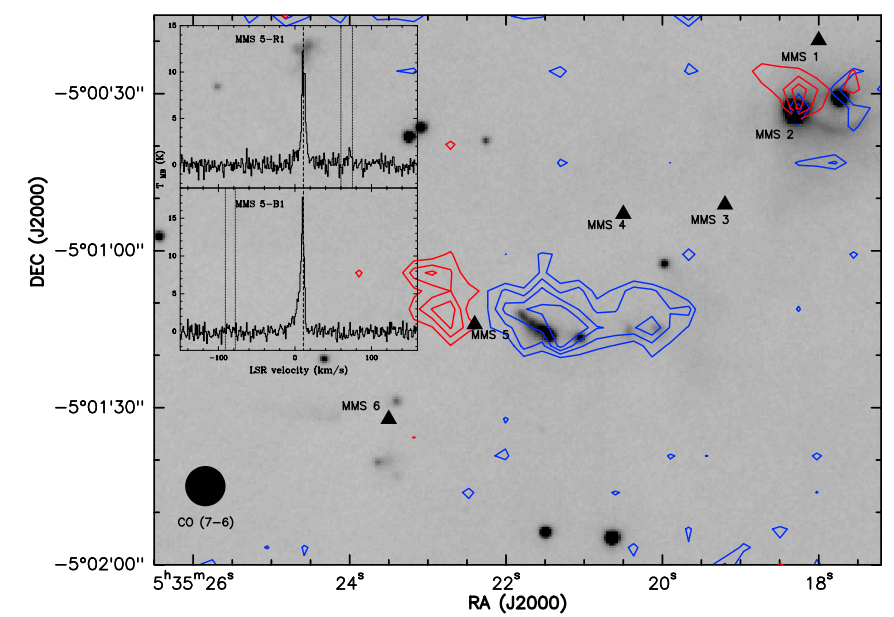

Fig. 7. CO (7-6) high-velocity emission map centered on MMS 5. Blue and red contours show the blue- and red-shifted high-velocity emission (integrated in the same velocity ranges as in Fig. 2), respectively. Contour spacing is $1 \sigma$, with $3 \sigma$ as the first contour $\left(\sigma=7.4 \mathrm{~K} \mathrm{~km} \mathrm{~s}^{-1}\right.$ for blue-shifted emission and $\sigma=6.7 \mathrm{~K} \mathrm{~km} \mathrm{~s}^{-1}$ for red-shifted emission). The background greyscale image is as in Fig. 4, the filled triangles as in Fig. 2. The inlay shows the CO (7-6) spectrum taken at the B1 and R1 positions, with the vertical lines as in Fig. 6.

The mass detection limit of our CO (6-5) observations, assuming a $T_{\text {ex }}$ of $60 \mathrm{~K}$ (following Table 6) and the rms at $1 \mathrm{~km} \mathrm{~s}^{-1}$, is $\sim 1 \times 10^{-5} M_{\odot}$ inside a beam of $9^{\prime \prime}$. For each outflow the $\mathrm{H}_{2}$ mass is reported in Table 7 . The total $\mathrm{H}_{2}$ masses range from $\sim 1 \times 10^{-2} M_{\odot}$ to $1 \times 10^{-3} M_{\odot}$. We note that our mass estimates based on the $\mathrm{CO}(6-5)$ emission are lower by a factor of 4 with respect to those reported in Table 3 of T08 for MMS 5, MMS 2, and FIR 6c (blue-lobe), while it is lower by a factor of 9 for FIR $6 \mathrm{~b}$ outflow. The higher discrepancy between the mass estimates from $\mathrm{CO}(3-2)$ and $\mathrm{CO}(6-5)$ in FIR 6 b outflow may be mostly due to the fact that our $\mathrm{CO}(6-5)$ map misses the upper
Table 5. CO integrated intensities at selected positions.

\begin{tabular}{lcccc}
\hline \hline Position & Velocity $\left(\mathrm{km} \mathrm{s}^{-1}\right)$ & \multicolumn{2}{c}{$T_{\mathrm{MB}} \mathrm{d} v(\sigma)\left(\mathrm{K} \mathrm{km} \mathrm{s}^{-1}\right)^{(a)}$} \\
\cline { 3 - 5 } & $\left(v_{\min }, v_{\max }\right)$ & $3-2$ & $6-5$ & $7-6$ \\
\hline FIR 6b-B1 & 0,8 & $32(2)$ & $17(1)$ & $<4^{(b)}$ \\
FIR 6b-R1 & 14,30 & $22(1)$ & $27(1)$ & $20(4)$ \\
FIR 6c-B1 & $-16,8$ & $23(1)$ & $27(2)$ & $20(5)$ \\
FIR 6c-B2 & $-10,8$ & $33(1)$ & $39(1)$ & $20(5)$ \\
MMS 5-B1 & $-25,7$ & $36(2)$ & $61(1)$ & $42(4)$ \\
EHV & $-95,-75$ & $<10^{(b)}$ & $11(1)$ & $3(3)$ \\
MMS 5-R1 & 16,31 & $6(2)$ & $16(1)$ & $8(2)$ \\
EHV & 60,75 & $<7^{(b)}$ & $14(1)$ & $8(2)$ \\
MMS 2-B1 & $-12,7$ & $16(2)$ & $18(1)$ & $12(3)$ \\
MMS 2-R1 & 16,30 & $9(1)$ & $11(1)$ & $8(3)$ \\
& 31,50 & $<8^{(b)}$ & $7(1)$ & $<3^{(b)}$ \\
\hline
\end{tabular}

Notes. Data convolved to 26" ASTE beam (i.e. CO (3-2) transition). ${ }^{(a)}$ Integrated between $v_{\min }, v_{\max }$. Statistical errors in parenthesis. ${ }^{(b)} 3 \sigma$ upper limit.

half of the red-shifted lobe, a region that was included in the $\mathrm{CO}$ (3-2) mass estimation from T08. By recomputing the mass from the $\mathrm{CO}(3-2)$ emission in an area equivalent to our $\mathrm{CO}$ (6-5) map, the difference is reduced to a factor of 3 . The lower masses obtained with respect to the $\mathrm{CO}$ (3-2) observations may be due the typical mass estimation uncertainties we already mention above; however, by looking at the maps it is clear in some cases (such as the MMS 2 outflow) that the emitting region in both lines is not exactly the same, with the CO (6-5) emission showing systematically more compact structures.

We also determine other parameters such as dynamical timescale $\left(t_{\mathrm{d}}\right)$, mass outflow rate $(\dot{M})$, mechanical force $\left(F_{\mathrm{m}}\right)$, kinetic energy $\left(E_{\mathrm{k}}\right)$, and mechanical luminosity $\left(L_{\mathrm{mech}}\right)$. These quantities are defined as follows: $t_{\mathrm{d}}=R / V, \dot{M}=M / t_{\mathrm{d}}$, 
A. I. Gómez-Ruiz et al.: Warm gas in protostellar outflows. II.

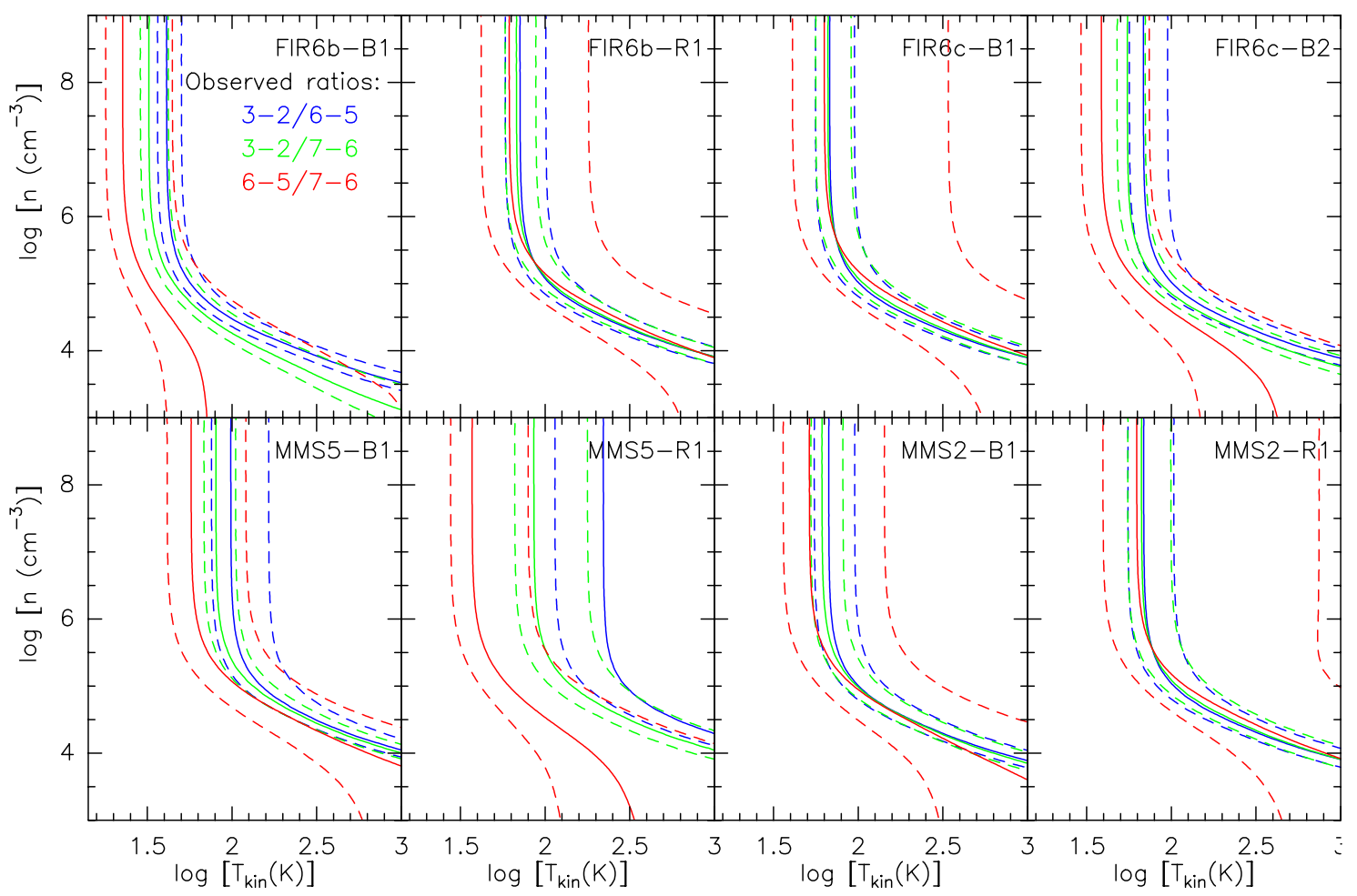

Fig. 8. Kinetic temperature $\left(T_{\text {kin }}\right)$ versus $\mathrm{H}_{2}$ density $(n)$ plots from LVG calculations. The curves show the observed (3-2)/(6-5), (3-2)/(7-6), and $(6-5) /(7-6)$ integrated intensity ratios (computed from Table 5). The dashed lines represent the errors in the line ratios. The positions are indicated by the labels in each panel.

Table 6. Rotational diagram.

\begin{tabular}{lll}
\hline \hline Position & $T_{\text {rot }}(\mathrm{K})$ & $N\left(10^{16} \mathrm{~cm}^{-2}\right)$ \\
\hline FIR 6b-B1 & $37 \pm 9$ & $1.5 \pm 0.8$ \\
FIR 6b-R1 & $69 \pm 4$ & $1.2 \pm 0.1$ \\
FIR 6c-B1 & $67 \pm 4$ & $1.2 \pm 0.1$ \\
FIR 6c-B2 & $60 \pm 10$ & $1.7 \pm 0.5$ \\
MMS 5-B1 & $83 \pm 12$ & $2.2 \pm 0.4$ \\
MMS 5-R1 & $95 \pm 65$ & $0.4 \pm 0.3$ \\
MMS 2-R1 & $68 \pm 5$ & $0.48 \pm 0.05$ \\
MMS 2-B1 & $63 \pm 4$ & $0.83 \pm 0.09$
\end{tabular}

Notes. We have used integrated intensities from Table 5. Statistical and calibration errors are taken into account.

$P=M \times \delta v_{\max }, \quad F_{\mathrm{m}}=M \times V / t_{\mathrm{d}}, \quad E_{\mathrm{k}}=M \times \delta v_{\max }^{2} / 2, \quad L_{\text {mech }}=E_{\mathrm{k}} / t_{\mathrm{d}}$ (e.g. Beuther et al. 2002). In the above expressions, $R=R_{\max } / \sin i$ and $V=\delta v_{\max } / \cos i$, where $R_{\max }$ and $\delta v_{\max }$ are the maximum extension and maximum relative velocity of the lobes, and $i$ the angle of the lobe with respect to the plane of the sky. To compare with T08 we assumed $i=45^{\circ}$ for all outflows. Table 7 shows our results. Here we also point out that considering the uncertainties in the mass estimation plus the geometry assumptions mentioned above, the kinematic parameters should be accurate within a factor of 10-30 (see, e.g., discussion in Cabrit \& Bertout 1990). Within the uncertainties mentioned above our results are consistent with T08.

\section{Discussion}

In the following we first discuss our data in comparison with other similar observations and after that focus on the case of the EHV emission from MMS 5.

\subsection{Comparison with other mid-J CO observations}

The outflow properties determined for our set of IM outflows are not very different from the values found by other authors in lowmass outflows (e.g., Paper I; Curtis et al. 2010). In particular, $F_{\mathrm{m}}$ and $E_{\mathrm{k}}$ values for the IM outflow MMS $5\left(3 \times 10^{-3} M_{\odot} \mathrm{km} \mathrm{s}^{-1}\right.$ $\mathrm{yr}^{-1}$ and $2 \times 10^{45} \mathrm{erg}$, respectively) are similar, within the typical errors, to those found in Paper I for the low-mass class 0 outflow L1448-mm $\left(\sim 8 \times 10^{-4} M_{\odot} \mathrm{km} \mathrm{s}^{-1} \mathrm{yr}^{-1}\right.$ and $2 \times 10^{45} \mathrm{erg}$, respectively). We therefore conclude that the IM outflow MMS 5 is as energetic as its low-mass counterpart in L1448-mm. However, we note that this may be only a particular case, since previous studies towards statistical significant samples have shown a trend of increasing energy and mechanical luminosity as the protostellar masses increase (e.g., Beuther et al. 2002).

van Kempen et al. (2016) presented CO (6-5) observations of a sample of six IM protostars. They determined outflow parameters employing methods similar to ours, allowing for a direct comparison. In general our results are in agreement with those of van Kempen et al. (2016). In particular, the outflow force is within the same range, except for the case of NGC 2071. More discrepant are the outflow masses which are higher for most of the sources in the van Kempen et al. (2016) sample. The latter is likely due to the larger size of the outflow lobes in that sample, which in turn may be an effect of the age. Given that we obtained a similar outflow force range as found for the van Kempen et al. (2016) sources that span a similar range of bolometric luminosities of the central objects, the correlation between the outflow force and the bolometric luminosity holds for both our sample and theirs.

\subsection{The EHV collimated jet from MMS 5}

Figures 7 and 6 show the $\mathrm{CO}(6-5)$ and $\mathrm{CO}(7-6)$ high-velocity and EHV emission, overlaid with the NIR emission from the 
Table 7. Outflow properties derived from CO (6-5).

\begin{tabular}{|c|c|c|c|c|c|c|c|}
\hline Velocity & $\begin{array}{l}\delta v_{\max }{ }^{(1)} \\
\left(\mathrm{km} \mathrm{s}^{-1}\right)\end{array}$ & $\begin{array}{c}R_{\max }(2) \\
\left(10^{-2}\right) \\
(\mathrm{pc})\end{array}$ & $\begin{array}{l}t_{\mathrm{d}}(3) \\
\left(10^{3}\right) \\
(\mathrm{yr})\end{array}$ & $\begin{array}{c}\mathrm{M}\left(\mathrm{H}_{2}\right)^{(4)} \\
\left(10^{-3}\right) \\
\left(M_{\odot}\right) \\
\end{array}$ & $\begin{array}{c}F_{\mathrm{m}}{ }^{(5)} \\
\left(10^{-5}\right) \\
\left(M_{\odot} \mathrm{km} \mathrm{s}^{-1} \mathrm{yr}^{-1}\right) \\
\end{array}$ & $\begin{array}{l}E_{\mathrm{k}}(6) \\
\left(10^{43}\right) \\
(\mathrm{erg}) \\
\end{array}$ & $\begin{array}{r}L_{\text {mec }}{ }^{(7)} \\
\left(L_{\odot}\right) \\
\end{array}$ \\
\hline \multicolumn{8}{|c|}{ FIR 6b } \\
\hline Blue & 8 & 3 & 4 & 6 & 2 & 0.7 & 0.02 \\
\hline Red & 19 & 4 & 2 & 3 & 3 & 2 & 0.07 \\
\hline Total & & & & 9 & 5 & 2.7 & 0.09 \\
\hline \multicolumn{8}{|c|}{ FIR 6c } \\
\hline Blue & 26 & 4 & 1 & 5 & 13 & 7 & 0.4 \\
\hline \multicolumn{8}{|c|}{ MMS 5} \\
\hline Blue & 106 & 6 & 0.6 & 9 & 230 & 200 & 28 \\
\hline $\operatorname{Red}$ & 59 & 4 & 0.6 & 5 & 70 & 30 & 5 \\
\hline Total & & & & 14 & 300 & 230 & 33 \\
\hline \multicolumn{8}{|c|}{ MMS 2} \\
\hline Blue & 23 & 4 & 2 & 1 & 2 & 1 & 0.06 \\
\hline Red & 19 & 4 & 2 & 0.4 & 0.5 & 0.3 & 0.01 \\
\hline Total & & & & 1.4 & 2.5 & 1.3 & 0.07 \\
\hline
\end{tabular}

Notes. ${ }^{(1)}$ Maximum relative velocity of the lobe. ${ }^{(2)}$ Maximum extension of the lobe. ${ }^{(3)}$ Dynamical time. ${ }^{(4)} \mathrm{H}_{2}$ mass. ${ }^{(5)}$ Mechanical force. ${ }^{(6)}$ Kinetic energy. ${ }^{(7)}$ Mechanical luminosity.

MMS 5 region. The main NIR features in this region are an extended monopolar structure and three knots to the west of it. These features are coincident with the $\mathrm{CO}(6-5)$ and $\mathrm{CO}$ (7-6) blue-shifted emission. On the other hand, no NIR structure is coincident with the red-shifted $\mathrm{CO}$ lobe, likely because of the known affect of extinction from the foreground gas in the red-shifted lobes (counter-jet) (see e.g., Noriega-Crespo \& Raga 2012). The NIR monopolar structure is indeed well collimated ( size $\sim 8.7^{\prime \prime} \times 1.7^{\prime \prime}$, hence a collimation factor $\sim 5$ ), which suggests the jet nature of the NIR emission. Since the knots to the west are not well aligned with this monopolar structure, it is possible that the jet from MMS 5 is precessing. As noted in Fig. 6, the EHV CO(6-5) blue-shifted emission seems to be coincident with the NIR collimated monopolar structure and the closest, brightest, NIR knot to the west of it. Therefore, the positional correlation with this NIR structure and knot may suggest the jet nature of the CO EHV emission. The higher angular resolution ALMA data reveal a collimated structure of the EHV gas in the $\mathrm{CO}(2-1)$ and $\mathrm{SiO}(5-4)$ transitions (Matsushita et al. 2019). The similar line profile and velocity range of the ALMA CO observations indicates that our mid-J CO observations are tracing the same outflow from MMS 5. However, the authors have found that the EHV gas and the NIR jet are not positionally coincident, suggesting that the $\mathrm{H}_{2}$ knots may not be related to the $\mathrm{CO}$ and $\mathrm{SiO}$ jets revealed by their data.

To our knowledge Cep-E and NGC 2071 are the only other confirmed cases of EHV emission from IM outflows (Lefloch et al. 2015; Gómez-Ruiz et al. 2012; Chernin \& Masson 1992; Hatchell et al. 1999), so the discovery of EHV gas from the MMS 5 outflow is an important addition to this kind of objects that would help to understand the jet phenomenon within the context of star formation theories, in particular for the IM case. Taking into account only the EHV emission and assuming that our CO (6-5) data resolved the major axis of the blue-shifted lobe, a dynamical timescale of $\sim 150 \mathrm{yr}$ is estimated for this kinematic structure, which is within the range measured in other very young IM outflows (e.g., Zapata et al. 2006). This result highlights the relevance of the mid- $J$ CO lines when searching for outflows in their early phase of evolution.

\section{Summary and conclusions}

The main results of this study on the mid- $J$ CO lines from IM outflows can be summarized in this way:

- The CO (6-5) (and in some cases the CO (7-6)) emission in OMC-2 FIR 6 and OMC-3 MMS 1-6 was found tracing outflows related to class 0-type IM objects within those regions.

- The CO (6-5) line profiles particularly highlight the secondary peaks, likely related to shock structures.

- Extremely high-velocity CO (6-5) emission was detected from the MMS 5 outflow in the OMC-3 region. The CO (7-6) transitions was detected at the $3 \sigma$ level from the red lobe of the same outflow.

- By comparison with previous observations, we found that at positions close to the central objects the $\mathrm{CO}(6-5)$ and (7-6) emission is stronger than the low $-J \mathrm{CO}(3-2)$ emission. This behavior is similar to what we found in low-mass outflows (L1448 and HH211).

- The kinematics and physical conditions of MMS 5 outflow suggest that it is the counterpart of the young class 0 lowmass outflows.

From these results we can conclude that the physical and kinematic properties of the young IM outflows presented here are similar to those found in outflows from Class 0 low-mass objects, and that given their excitation requirements, the mid- $J$ CO lines are important tracers of young outflows likely related to jets.

Acknowledgements. We wish to thank all the APEX staff in Chile for their enthusiastic help during these observations. AIGR was supported through a stipend 
A. I. Gómez-Ruiz et al.: Warm gas in protostellar outflows. II.

from the International Max-Planck Research School for Astronomy and Astrophysics at the universities of Bonn and Cologne, and is currently supported by CONACYT under the program Cátedras CONACYT para jóvenes investigadores. A.G. acknowledges support via the grant ANR-09-BLAN-0231-01 from the French Agence Nationale de la Recherche as part of the SCHISM project.

\section{References}

Aso, Y., Tatematsu, K., Sekimoto, Y., et al. 2000, ApJS, 131, 465

Bachiller, R., Martin-Pintado, J., Tafalla, M., Cernicharo, J., \& Lazareff, B. 1990 A\&A, 231, 174

Beltrán, M. T. 2015, Ap\&SS, 355, 283

Beltrán, M. T., Estalella, R., Girart, J. M., Ho, P. T. P., \& Anglada, G. 2008, A\&A, 481, 93

Beuther, H., Schilke, P., Sridharan, T. K., et al. 2002, A\&A, 383, 892

Cabrit, S., \& Bertout, C. 1990, ApJ, 348, 530

Chernin, L. M., \& Masson, C. R. 1992, ApJ, 396, L35

Chini, R., Reipurth, B., Ward-Thompson, D., et al. 1997, ApJ, 474, L135

Crimier, N., Ceccarelli, C., Alonso-Albi, T., et al. 2010, A\&A, 516, A102

Curtis, E. I., Richer, J. S., Swift, J. J., \& Williams, J. P. 2010, MNRAS, 408, 1516

Fuente, A., Neri, R., Martín-Pintado, J., et al. 2001, A\&A, 366, 873

Fuente, A., Ceccarelli, C., Neri, R., et al. 2007, A\&A, 468, L37

Furlan, E., Fischer, W. J., Ali, B., et al. 2016, ApJS, 224, 5

Gómez-Ruiz, A. I., Gusdorf, A., Leurini, S., et al. 2012, A\&A, 542, L9

Gómez-Ruiz, A. I., Wyrowski, F., Gusdorf, A., et al. 2013, A\&A, 555, A8

Güsten, R., Nyman, L. A., Schilke, P., et al. 2006, A\&A, 454, L13

Hatchell, J., Fuller, G. A., \& Ladd, E. F. 1999, A\&A, 346, 278

Johnstone, D., \& Bally, J. 1999, ApJ, 510, L49

Kahane, C., Jaber Al-Edhari, A., Ceccarelli, C., et al. 2018, ApJ, 852, 130

Kasemann, C., Güsten, R., Heyminck, S., et al. 2006, SPIE Conf. Ser., 6275, 19

Klein, B., Philipp, S. D., Krämer, I., et al. 2006, A\&A, 454, L29

Kounkel, M., Covey, K., Suárez, G., et al. 2018, AJ, 156, 84
Lefloch, B., Gusdorf, A., Codella, C., et al. 2015, A\&A, 581, A4

Matsushita, Y., Takahashi, S., Machida, M. N., \& Tomisaka, K. 2019, ApJ, 871, 221

Noriega-Crespo, A., \& Raga, A. C. 2012, ApJ, 750, 101

Schöier, F. L., van der Tak, F. F. S., van Dishoeck, E. F., \& Black, J. H. 2005, A\&A, 432, 369

Shimajiri, Y., Takahashi, S., Takakuwa, S., Saito, M., \& Kawabe, R. 2009, PASJ, 61,1055

Stanke, T., McCaughrean, M. J., \& Zinnecker, H. 2002, A\&A, 392, 239

Takahashi, S., \& Ho, P. T. P. 2012, ApJ, 745, L10

Takahashi, S., Saito, M., Takakuwa, S., \& Kawabe, R. 2006, ApJ, 651, 933

Takahashi, S., Saito, M., Ohashi, N., et al. 2008, ApJ, 688, 344

Takahashi, S., Ho, P. T. P., Tang, Y.-W., Kawabe, R., \& Saito, M. 2009, ApJ, 704, 1459

Takahashi, S., Saigo, K., Ho, P. T. P., \& Tomida, K. 2012, ApJ, 752, 10

Takahashi, S., Ho, P. T. P., Teixeira, P. S., Zapata, L. A., \& Su, Y.-N. 2013, ApJ, 763,57

Tsujimoto, M., Koyama, K., Kobayashi, N., et al. 2004, PASJ, 56, 341

van der Tak, F. F. S., Black, J. H., Schöier, F. L., Jansen, D. J., \& van Dishoeck, E. F. 2007, A\&A, 468, A627

van Kempen, T. A., Longmore, S. N., Johnstone, D., Pillai, T., \& Fuente, A. 2012, ApJ, 751, 137

van Kempen, T. A., Hogerheijde, M. R., van Dishoeck, E. F., et al. 2016, A\&A, 587, A 17

Williams, J. P., Plambeck, R. L., \& Heyer, M. H. 2003, ApJ, 591, 1025

Wilson, T. L., \& Matteucci, F. 1992, A\&ARv, 4, 1

Yang, B., Stancil, P. C., Balakrishnan, N., \& Forrey, R. C. 2010, ApJ, 718, 1062

Yildız, U. A., Kristensen, L. E., van Dishoeck, E. F., et al. 2015, A\&A, 576, A109

Yu, K. C., Bally, J., \& Devine, D. 1997, ApJ, 485, L45

Zapata, L. A., Rodríguez, L. F., Kurtz, S. E., \& O’Dell, C. R. 2004, AJ, 127, 2252

Zapata, L. A., Rodríguez, L. F., Ho, P. T. P., et al. 2005, ApJ, 630, L85

Zapata, L. A., Ho, P. T. P., Rodríguez, L. F., et al. 2006, ApJ, 653, 398 